\title{
A case of indirect carotid cavernous fistula presenting with proptosis and pulsatile exophthalmos
}

\author{
(1) Gökhan Yüce', (1) Adem Doğan² \\ 1 University of Health Sciences Turkey, Gülhane Training and Research Hospital, Clinic of Radiology, Ankara, Turkey \\ 2University of Health Sciences Turkey, Gülhane Training and Research Hospital, Clinic of Neurosurgery, Ankara, Turkey
}

Date submitted:

28.05.2020

Date accepted:

15.06.2020

Online publication date:

15.06.2021

\section{Corresponding Author:}

Gökhan Yüce, M.D., University of

Health Sciences Turkey, Gülhane

Training and Research Hospital, Clinic of Radiology, Ankara, Turkey

ORCID:

orcid.org/0000-0003-3546-7965

Keywords: Flow diverter stent, carotid cavernous fistula, CCF superior

ophthalmic vein

\begin{abstract}
Carotid cavernous fistulas (CCF) are abnormal arteriovenous anastomoses between the internal carotid artery (ICA) and the cavernous sinus (CS). There are two broad categories of CCFs with different clinical presentations in direct and indirect form. Internal carotid artery and the CS in the "direct" or "high flow" CCF, and branches of the internal and external carotid artery in "indirect" or "low flow" CCF are found abnormally connected. In the present case, a CCF draining into the ophthalmic vein was detected between the ICA and the CSs. An endovascular flow diverter stent was inserted, and the fistula line was closed. The complaints of the patient immediately disappeared. In CCFs, the flowing stent can completely close the fistula line in a selected patient and appropriate localization.
\end{abstract}

\section{Introduction}

Carotid cavernous fistulas (CCF) are abnormal arteriovenous anastomoses between the internal carotid artery (ICA) and the cavernous sinus (CS). CCFs can be classified as spontaneous or traumatic according to the etiology and direct or indirect (dural) according to the anatomical features (1). In a direct CCF, arterial blood flows to the CS through the shunt in the intracavernous part of the ICA, causing high blood flow and high pressure. On the other hand, indirect (dural) CCFs result from the connection of the meningeal branches of the ICA with the CS, causing low blood flow and low pressure $(1,2)$. Intracranial dural arteriovenous fistulas account for 10 to $15 \%$ of all intracranial arteriovenous malformations (3). Proptosis, ophthalmoplegia, headache, eyeball, sclera, and redness of conjunctiva may be observed. Depending on whether the indirect fistulas are drained anteriorly or posteriorly, different clinical manifestations may occur. In the treatment of cavernous carotid sinuses, endovascular flow converting stents may improve the clinical findings when used in a selected patient and appropriate anatomical location.

\section{Case Presentation}

A 58-year-old female patient presented to the ophthalmology clinic with a complaint of redness in her left eye for one month. Due to her prolonged complaints and neurological examinations, CCF was suspected. Digital substraction angiography revealed a low-flow indirect (type B) CCF associated with the CSs at the level of petrous and cavernous segments of the internal cerebral artery (segments C2-C3). No additional treatment was considered. The patient was re-admitted because of proptosis three months later. An urgent angiography showed increased 
blood flow to the fistula, and an aggravated filling pattern in the left superior ophthalmic vein (SOV) showed (Figure 1). Neurological examination revealed pulsatile exophthalmos, proptosis, and conjunctival hyperemia. There was no systemic disease or trauma in the history of the patient. A flow diverter stent was placed to cover the localization of the branches feeding the fistula from the ICA. The stent was correctly opened. By the $3^{\text {rd }}$ month, control angiography showed the fistula line was closed entirely (Figure 2), and exophthalmos and proptosis complaints resolved completely.

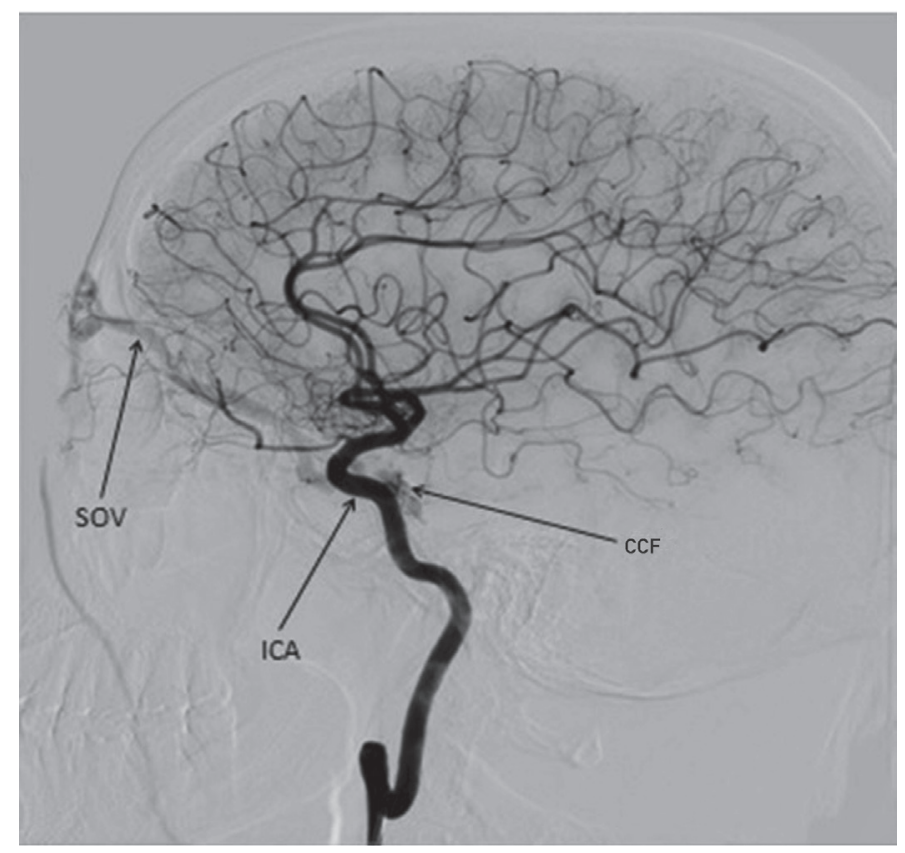

Figure 1. Preoperative angiographic image

SOV: Superior ophthalmic vein, ICA: Internal carotid artery, CCF: Carotid cavernous fistula

\section{Discussion}

CCF is the result of an abnormal connection between ICA and CS. CCFs can be classified according to etiology (spontaneous or traumatic), flow velocity (low or high flow velocity), or angiographic structure (direct or indirect) $(1,2,4)$. Barrow et al. (1) are divided CCFs into four groups based on pathogenesis and arterial supply. In type A, also referred to as direct CCF, arterial blood passes through the shunt in the intracavernous part of the ICA to the CS, where high blood flow and high pressure are formed. Connections occur between the CS and dural branches of ICA in type B fistulas, CS and dural branches of the external carotid artery (ECA) in type $C$ fistulas, $C S$ and dural branches of both ICA and ECA in type D fistulas. Type B, C, D fistulas are indirect (dural) CCFs, and these fistulas are characterized by low flow and low pressure $(1,2,4,5)$.

The classic presentation of direct, high-flow CCFs is the sudden onset of symptoms due to the direct passage of highpressure arterial blood into the CS and ophthalmic vein and

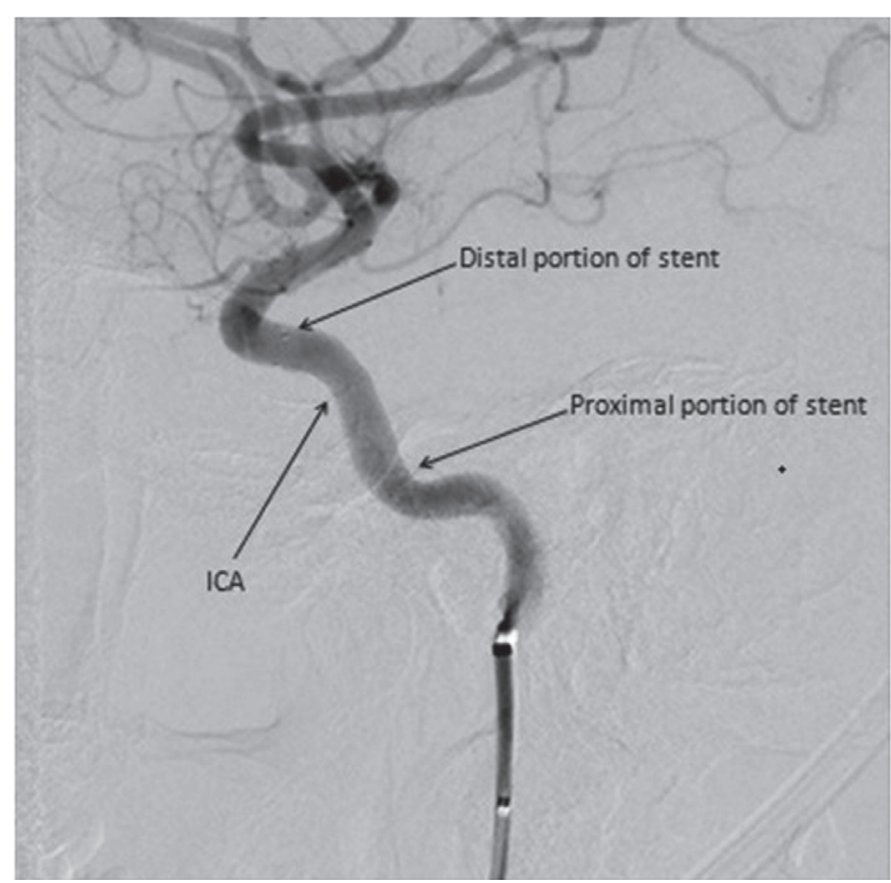

Figure 2. Postoperative third month (after flow diverter stent) angiographic image

ICA: Internal carotid artery

venous hypertension $(2,4)$. Clinical findings in indirect CCFs are similar to the direct fistulas when drained from the anterior to superior and inferior ophthalmic veins, but the results are milder in indirect CCFs because of slow blood flow. Miller described the clinical manifestations of indirect CCFs based on whether the indirect fistulas drain into the anterior (superior and inferior ophthalmic veins) or posterior (superior and inferior petrosal sinuses) (6). Proptosis, congestion, and orbital murmur are not seen in the posteriorly drained fistulas. Therefore, they are defined as white-eyed shunts (7). The oculomotor nerve is most commonly affected $(4,5,8-10)$. Most ocular congestion findings are absent, and unilateral orbital pain may occur. In our patient, we observed drainage into the SOV.

The clinical manifestations are milder in indirect CCFs $(2,4,5)$. Conjunctival redness is the most prominent feature and can be misdiagnosed as conjunctivitis, episcleritis, or thyroid disease. Other findings include chemosis, proptosis, diplopia and ophthalmoparesis, retroorbital pain, increased intraocular pressure, and decreased visual acuity $(2,5)$. Indirect CCFs often develop spontaneously, and spontaneous CCFs usually occur in the elderly and women. It may occur secondary to ruptured cavernous ICA aneurysms.

CCFs occur due to arterial wall defects after minor stress such as valsalva and cough in patients with a collagen vascular disease such as fibromuscular dysplasia and Ehler-Danlos syndrome. Arterial hypertension, atherosclerotic vascular disease, pregnancy, minor trauma, strains, and diabetes are among the predisposing causes $(2,4)$. 
CT angiography, magnetic resonance angiography, orbital ultrasonography, transorbital, and transcranial color Doppler imaging may be helpful in diagnosis. The gold standard diagnostic test is a selective angiography of bilateral ICAs and ECAs in all types of CCF. Treatment decision in CCF is dependent primarily on drainage pattern and symptoms $(2,4,5)$. It is necessary to determine the exact location of the fistula in both types of CCFs and to identify the vessels and drainage pattern feeding the fistula before treatment. The most effective treatment is the obliteration of arterial-venous fistula connection through endovascular intervention and surgery. Another potential treatment option is stereotactic radiotherapy.

\section{Conclusion}

The patient was admitted with complaints of exophthalmos and proptosis, and angiography was performed to evaluate the risk of an aneurysm or other vascular pathology. An indirect CCF was identified, and a flow diverter stent was placed to close the fistula line.

\section{Acknowledgments}

We would like to thank the staff of Gülhane Training and Research Hospital, Clinics of Neurosurgery and Radiology, who contributed to the writing of this article.

\section{Ethics}

Informed Consent: Written, informed consent was obtained from the patient for this study.

Peer-review: Externally peer-reviewed.

\section{Authorship Contributions}

Surgical and Medical Practices: G.Y., Concept: A.D., Design: A.D., Data Collection or Processing: G.Y., A.D., Analysis or Interpretation: A.D., Literature Search: G.Y., A.D., Writing: G.Y., A.D.

Conflict of Interest: No conflict of interest was declared by the authors.
Financial Disclosure: The authors declared that this study received no financial support.

\section{References}

1. Barrow DL, Spector RH, Braun IF, Landman, et al. Classification and treatment of spontaneous carotidcavernous sinus fistulas. J Neurosurg. 1985;62:248256.

2. Ellis JA, Goldstein H, Sander Connolly Jr E, et al. Carotidcavernous fistulas. Neurosurg Focus. 2012;32:111.

3. Newton $\mathrm{TH}$, Cronqvist S. Involvement of dural arteries in intracranial arteriovenous malformations. Radiology. 1969;93:1071-1078.

4. Gruman AJ, Boivin-Faure L, Chapot R, et al. Ophthalmologic outcome of direct and indirect carotid cavernous fistulas. Int Ophthalmol. 2012;32:153-159.

5. Miller NR. Diagnosis and management of dural carotidcavernous sinus fistulas. Neurosurg Focus. 2007;23:1-15.

6. Miller NR. Dural carotid-cavernous fistulas: epidemiology, clinical presentation and management. Neurosurg Clin N Am. 2012;23:179-192.

7. Acierno MD, Trobe JD, Cornblath WT, Gebarski SS. Painful oculomotor palsy caused by posterior-draining dural carotid cavernous fistulas. Arch Ophthalmol. 1995;113:1045-1049.

8. Miyachi S. Negoro M. Handa T, et al. Dural carotid cavernous sinus fistula presenting as isolated oculomotor nerve palsy. Surg Neurol. 1993;39:105-109.

9. Wu HC, Ro LS, Chen CJ, et al. Isolated ocular motor nerve palsy in dural carotid-cavernous sinus fistula. Eur J Neurol. 2006;13:1221-1225.

10. Selky AK, Purvin VA. Isolated trochlear nerve palsy secondary to dural carotid-cavernous sinus fistula. J Neuroophthalmol. 1994;14:52-54. 